\title{
Exploiting non-equilibrium phase separation for self-assembly
}

\section{Citation}

Grünwald, Michael, Simon Tricard, George M. Whitesides, and Phillip L. Geissler. 2016.

Exploiting Non-Equilibrium Phase Separation for Self-Assembly. Soft Matter 12, no. 5: 15171524. doi:10.1039/c5sm01922b.

\section{Published Version}

10.1039/c5sm01922b

\section{Permanent link}

http://nrs.harvard.edu/urn-3:HUL.InstRepos:29991616

\section{Terms of Use}

This article was downloaded from Harvard University's DASH repository, and is made available under the terms and conditions applicable to Open Access Policy Articles, as set forth at http:// nrs.harvard.edu/urn-3:HUL.InstRepos:dash.current.terms-of-use\#OAP

\section{Share Your Story}

The Harvard community has made this article openly available.

Please share how this access benefits you. Submit a story.

Accessibility 


\section{Exploiting non-equilibrium phase separation for self-assembly}

Michael Grünwald, ${ }^{1}$ Simon Tricard, ${ }^{2}$ George M. Whitesides, ${ }^{2}$ and Phillip L. Geissler ${ }^{3}$

1) Department of Chemistry, University of Utah, Salt Lake City, Utah 84112

${ }^{2)}$ Department of Chemistry and Chemical Biology, Harvard University, 12 Oxford Street, Cambridge, MA 02138

3) Department of Chemistry, University of California, Berkeley, California 94720

Demixing can occur in systems of two or more particle species that experience different driving forces, e.g., mixtures of self-propelled active particles or of oppositely charged colloids subject to an electric field. Here we show with macroscopic experiments and computer simulations that the forces underlying such non-equilibrium segregation can be used to control the self-assembly of particles that lack attractive interactions. We demonstrate that, depending on the direction, amplitude and frequency of a periodic external force acting on one particle species, the structures formed by a second, undriven species can range from compact clusters to elongated, string-like patterns. 


\section{INTRODUCTION}

Systems of particles that are driven far from equilibrium can self-assemble into patterns that are much more complex than their typical equilibrium structures. Examples of such patterns occur in many living systems and range from flocking birds to "swimming" bacteria to actin filament networks. ${ }^{1}$ Building similarly complex patterns with synthetic nanoparticles that are driven out of equilibrium is a promising route towards functional and responsive nanomaterials. ${ }^{2}$ However, mimicking the targeted energy input on the level of single nanoparticles, which is a hallmark of self-assembly in living systems, remains a formidable challenge.

Recent experimental and theoretical results show that long-wavelength spatial patterns can been achieved in multicomponent systems that are driven out of equilibrium in much simpler ways. In particular, mixtures of oppositely charged colloids on a substrate demix into striped patterns when an external electric AC field is applied. ${ }^{3,4}$ Different patterns can be obtained by changing the frequency of the electric field.$^{5-7}$ Similar demixing behavior has been observed in granular systems under vibration and related model systems. ${ }^{8,9}$

In this paper we examine the possibility of exploiting this segregation tendency, for the purpose of preparing non-equilibrium states that are patterned on much smaller scales. We do so using both experiment and computer simulation. Our experimental systems comprise macroscopic plastic solids, whose motions in a container are made roughly thermal in nature through agitation. However, depending on the shape of the solids, agitation results in different dynamics with respect to the container. We show that sufficiently strong amplitudes and suitable frequencies of agitation lead to segregation and self-assembly by particle shape. Our simulations explore the behavior of very similar model systems, but leverage the computational ease of varying particle shape, dimensionality of the system, and the nature of agitation. We analyze the effective attractive forces induced by agitation and show that by changing the driving force and particle shape, the resulting self-assembled structures can be switched from simple compact aggregates to patterns that reflect the directionality of the driving force. 


\section{DIFFERENTIAL "SHAKING" CAN INDUCE ASSEMBLY OF NON-ATTRACTING PARTICLES}

Mechanical agitation of macroscopic objects can induce random motion that resembles the equilibrium dynamics of microscopic particles. ${ }^{10,11}$ In particular, agitation can lead to the formation of patterns that correspond to equilibrium states of thermal systems. ${ }^{12-15}$ For instance, randomly packed beads crystallize in an agitated container, and oppositely charged plastic shapes form binary crystal lattices under shaking. ${ }^{16-18}$. However, thermal equilibrium imposes strict rules on the statistics of energy fluctuations that can be easily violated by mechanical agitation. In particular, random thermal forces acting on different particles are statistically independent. Mechanical agitation, on the other hand, introduces correlations in the dynamics of different particles that can lead to dramatic deviations from equilibrium behavior. $^{19}$

Our experimental setup, illustrated in Figure 1a, exemplifies this situation. It consists of a shallow, circular container that is connected to two motors for quasi-random agitation (see Methods section for details). ${ }^{20}$ The container is filled with plastic solids: a large number of spherical beads, six triangular prisms ("wedges"), and one star-shaped object. The solids lack any noticeable attractive interactions, as discussed in the Methods section. Figure 1b shows a time series of snapshots of the system as it is agitated by the motor. Within 13 minutes, wedges and star aggregate and assemble into a compact unit.

The assembled state observed in our experiment is not caused by depletion attraction. Such an entropic bias can, in principle, drive phase separation in mixtures of particles with different size. ${ }^{21,22}$ To show that this mechanism does not apply in our case, we performed Monte Carlo computer simulations of a two-dimensional system of hard particles with shapes similar to the ones used in the experiment (see Methods section for simulation details). First, we performed a number of simulation runs of the system in thermal equilibrium, i.e., with standard Metropolis dynamics that sample the Boltzmann distribution of states. If the assembly found in experiment were a signature of the system relaxing towards equilibrium, the same aggregation should be observable in the Monte Carlo simulation. However, no aggregation was observed. To the contrary, as illustrated in a series of snapshots in Fig. 1c, the assembled state was not even found to be metastable: when the system was prepared in the assembled state, it quickly dispersed. ${ }^{23}$ 
a

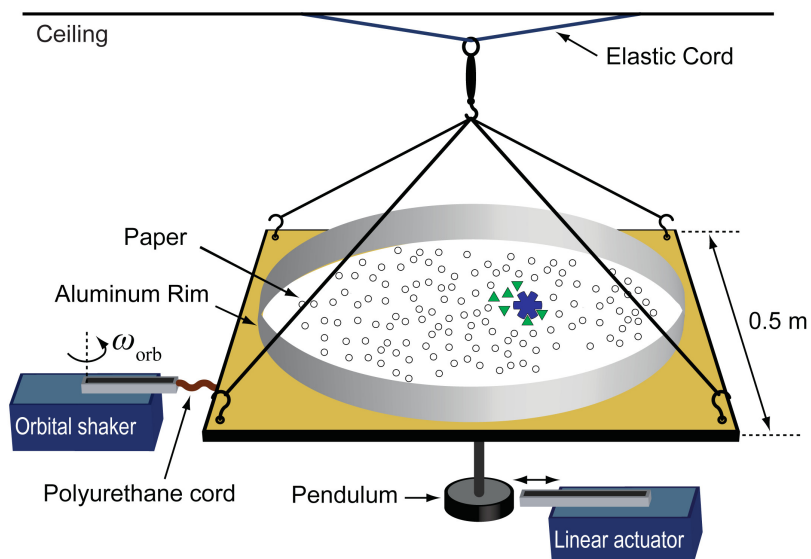

b

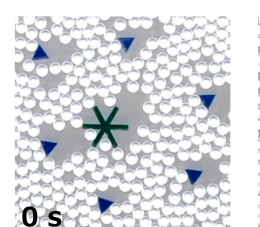

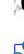
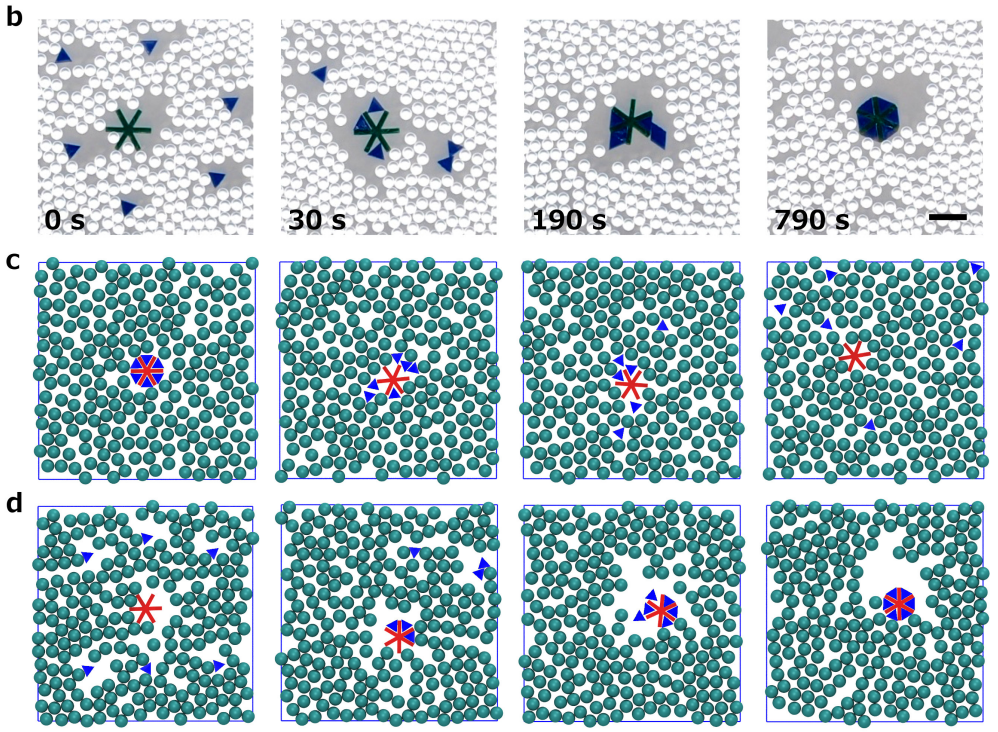

e
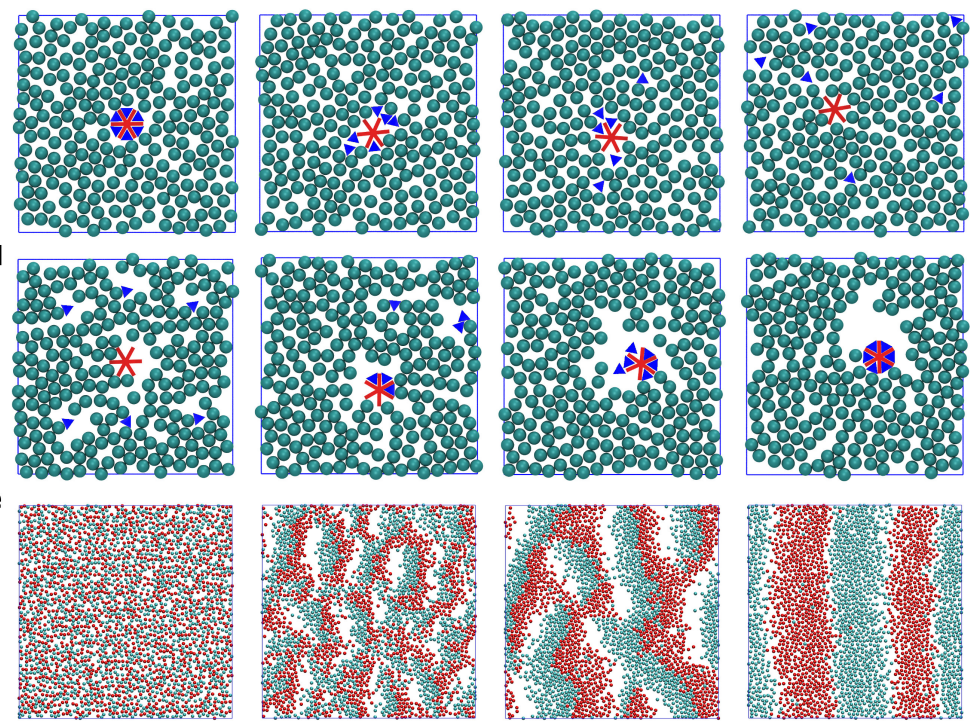

FIG. 1. Agitation induced self-assembly. (a). Experimental setup. (b) Time series of photographs of the experimental system under agitation, looking down onto the paper plain. (Scale bar $=2 \mathrm{~cm}$.) (c) Time series of snapshots from an equilibrium Monte Carlo simulation of hard shapes. Initiated as a compact unit, the star and wedges disperse. (d) Snapshots from a Monte Carlo simulation with biased displacement moves of discs. Initiated in a dispersed state, star and wedges aggregate and assemble. Note the void regions around star and wedges in both simulations and experiment, which form due to the slower motion of these shapes compared to discs/beads. (e) Snapshots from a Langevin molecular dynamics simulation of a binary mixture of soft discs, driven by a periodic external force. Blue colored particles experience stronger friction forces. 
Self-assembly can be reproduced in simulations, however, by including the different effective forces imparted by the agitation on different solids. The beads in the experiment roll and thus move in the frame of reference of the container when it is agitated. The flat bottom faces of the star and wedges, on the other hand, effect large static friction forces. In fact, when the container is agitated, these shapes will only move as a result of collisions with beads. To model this asymmetry in the dynamics of the different shapes, we performed Monte Carlo simulations that employed the following particle moves: Displacements of discs (with diameter $\sigma$ ) were drawn from a uniform distribution with zero mean, a customary choice in equilibrium Monte Carlo simulations. Displacements of wedges and star, however, were drawn from time-dependent distributions centered on a vector $\mathbf{a}(t)$ with components $a_{x}(t)=a \cos (2 \pi t / \tau)$ and $a_{y}(t)=a \sin (2 \pi t / \tau)$, where $t$ is time, and $a$ and $\tau$ are the magnitude and rotational period of the average displacement vector. These particle displacement rules mimic the effects of the circular motion of the orbital motor used to agitate the experimental setup. More importantly, they violate microscopic reversibility of the dynamics and result in a non-Boltzmann distribution of states. For a broad range of values of the parameters $a$ and $\tau$, we observe segregation and self-assembly of star and wedges, as illustrated in Figure 1d. (In this case, we used $a=0.6 \sigma$ and $\tau=1200$ sweeps.) Very similar results were obtained from simulations that apply the periodic bias to the beads, rather than to the star and wedges, suggesting that the choice of reference frame is qualitatively unimportant to the dynamics of assembly.

\section{SEGREGATION IS CAUSED BY ASYMMETRICAL COLLISION RATES}

Our computer simulations show that such nonequilibrium segregation is not limited to the special case of sinusoidal biases, but can occur very generally when drift velocity, induced by an external field, differs substantially between two or more particle types. ${ }^{5}$ In particular, concentrating on the Langevin dynamics of dense fluids consisting of two particle types $(A$ and $B$ ), segregation can be observed in the following scenarios:

- One of the particle types is driven by a periodic driving force.

- $A$ and $B$ experience the same external periodic force, but have different friction coef- 


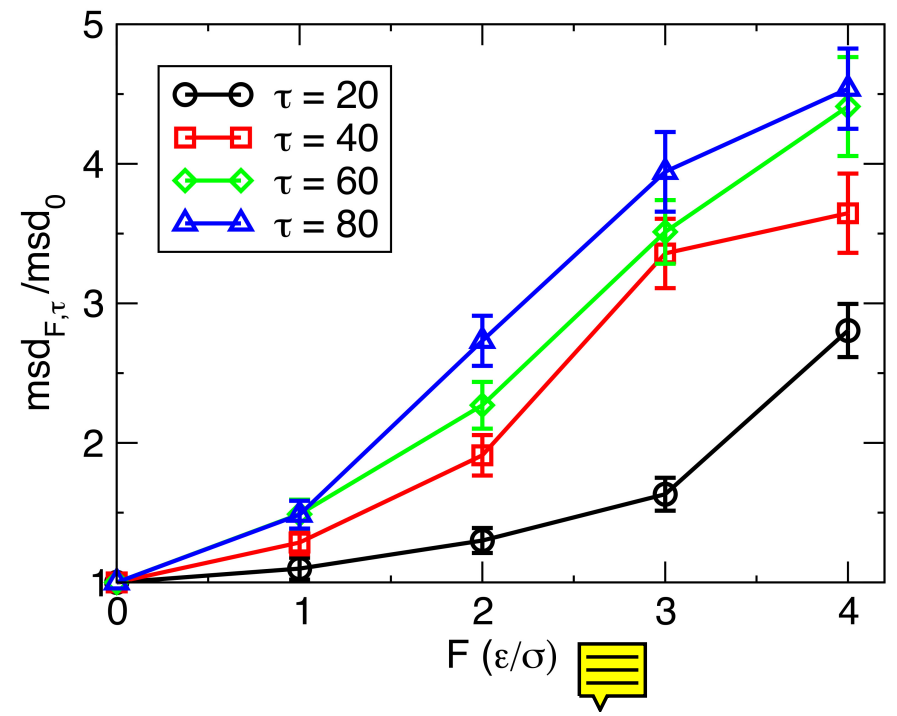

FIG. 2. Shaking causes particle mobilities that depend on local environment. Mean squared displacement of a single undriven particle in a bath of driven particles, as a function of amplitude $F$ and period $\tau$ (in units of $\sigma \sqrt{m / \epsilon}$ ) of the periodic external force. Results are normalized by the mean squared displacement at zero driving, i.e., by the mobility of a particle in a homogeneous environment of its own type. All data points reflect averages over at least 150 independent trajectories. Error bars indicate one standard deviation.

ficients. (See Figure 1e for snapshots of the segregation process in this case.)

- $A$ and $B$ experience different, non-periodic driving forces.

In all the cases described above, particles have collision rates that depend on the composition of their immediate environment: Particles that are surrounded by their own type have lower collision rates than particles in heterogeneous environments. For illustration purposes, consider the effect of an arbitrary, time-dependent external force $\mathbf{F}(t)$ that acts on all particles in a dense system of identical particles. In this case, the equations of motion for the relative positions of particles are unchanged by $\mathbf{F}(t)$; collision rates are thus independent of $\mathbf{F}(t)$, too. Consider now the case where $\mathbf{F}(t)$ acts on only one of two particle types in a mixture of otherwise identical particles. Relative motion of unlike particles is modulated by $\mathbf{F}(t)$ in this case, and particles in environments of heterogeneous composition experience comparatively large numbers of collisions. (See Supporting Information for a discussion of the correspondence between the dynamics of particles subject to mechanical agitation and direct external forces.) 
Differences in collision rates directly translate into different particle mobilities. To illustrate this point, we calculate mean squared displacements of particles in environments of different composition. We first follow numerically the Langevin dynamics of a dense fluid of particles that are driven by a periodic external force $\mathbf{F}(t)$ with components $F_{x}=F \cos (2 \pi t / \tau)$ and $\left.F_{y}=F \sin (2 \pi t / \tau)\right)$. These particles, symmetrically biased by the driving, have a mean square displacement that is independent of amplitude $F$ and period $\tau$ of the driving. In particular, since $\int_{0}^{\tau} d t \mathbf{F}(t)=0$, the driving force does not effect a net translation over long times and the mean square displacement is equal to that measured in the undriven system (i.e, for $F=0$ ), which we refer to as $m_{s} d_{0}$. By contrast, the mobility of a single undriven particle, added to the driven system, depends sensitively on the driving force. In Figure 2 , we plot the mean squared displacement of this particle $\left(m s d_{F, \tau}\right)$ as a function of $F$ for several values of $\tau$, normalized by $m s d_{0}$. We observe a marked increase in the mobility of the particle with both $F$ and $\tau$.

The net effect of these disparate mobilities is a particle flux away from regions of heterogeneous composition, which can lead to phase separation. ${ }^{24,25}$ This mechanism is also responsible for pattern formation in model systems of "active" particles, i.e., particles with

intrinsic mobility. ${ }^{25-31}$. Corté and coworkers have used an analogous argument to rationalize the transformation from an active to an inactive state in computer models of colloidal suspensions under periodic shear ${ }^{32}$.

\section{THE SPATIAL STRUCTURE OF SEGREGATED PHASES CAN BE CONTROLLED}

We now turn our attention to controlling the patterns formed within the segregated phases. In our experiments, we observe that the extent to which star and wedges assemble into a compact unit depends on the compatibility of the two shapes. Figure $3 \mathrm{~b}$ shows snapshots from an experiment that is identical to the one presented in Figure 1 except for the star-shaped solid, which in this case has smaller pockets. While aggregation of star and wedges occurs also in this case, only incomplete assembly is observed. Simulations of similarly modified shapes also show incomplete assembly — but furthermore demonstrate that the degree of assembly can be tuned via the amplitude of the driving force. In Figure 3a we plot the average number $n_{\mathrm{g}}$ of aggregated wedges (i.e., the number of wedges in the 


\section{a}

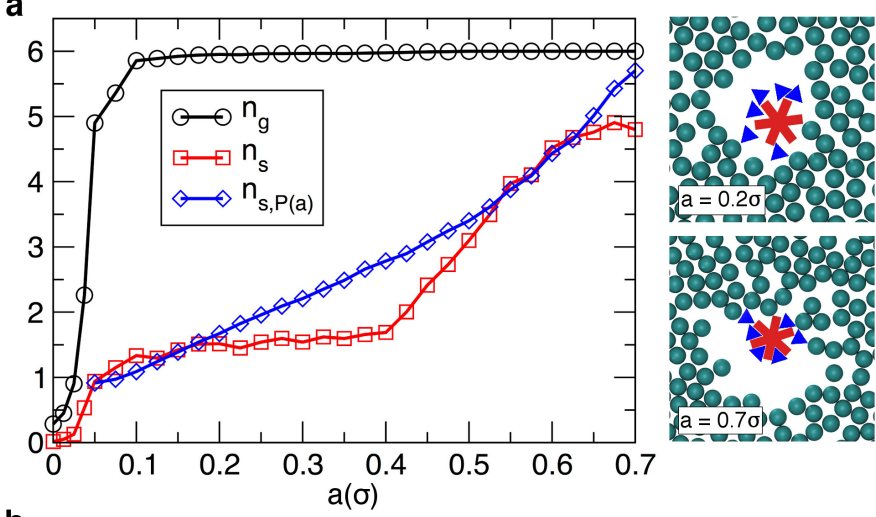

b

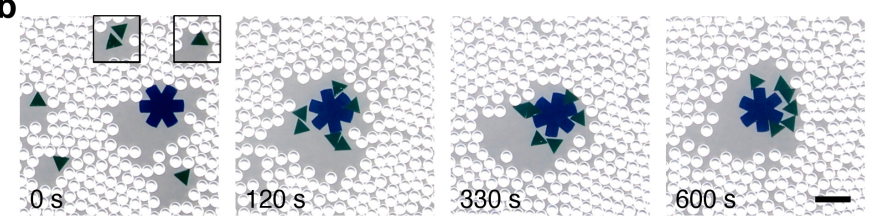

FIG. 3. Shaking amplitude controls an effective pressure. (a) Number of aggregated wedges $n_{\mathrm{g}}$ and assembled wedges $n_{\mathrm{s}}$ as a function of driving amplitude $a$ in Monte Carlo simulations of the star-and-wedge system at $\tau=1200$ sweeps. Snapshots show two typical configurations. Equilibrium simulations at constant pressure $P$ show similar degrees of assembly $n_{\mathrm{s}, P(a)}$, when $P$ is mapped onto $a$ as described in the text. (b) Snapshots of experimental system show aggregation, but no assembly, when the pockets of the star-shaped object were made smaller. (Insets in the first snapshot show wedges located outside of the field of view of the camera.)

immediate vicinity of the star) and the average number $n_{\mathrm{s}}$ of assembled wedges (i.e., the number of wedges occupying a pocket of the star) as a function of the amplitude of the driving. We observe three regimes, though not sharply delimited: For $a<0.1 \sigma$, we observe only partial segregation of shapes, indicated by values of $n_{\mathrm{g}}<6$. (At $a=0$, the system is at equilibrium.) For intermediate values of $0.1 \sigma<a<0.4 \sigma$, we observe essentially complete segregation of shapes, but little assembly $\left(n_{\mathrm{s}} \approx 1.5\right.$ in this regime). Finally, for large values of $a>0.4 \sigma$, the number of assembled wedges gradually increases with $a$ until almost complete assembly is obtained.

The progression of states observed for star and wedges with increasing amplitude of driving - dilute vapor, disordered aggregate, ordered aggregate - bears similarity with familiar equilibrium phase behavior at conditions of increasing pressure. Our computer simulations indeed show that the basic influence of an environment of "shaking" particles is to 
generate an effective pressure on a segregated phase of unbiased particles. ${ }^{33}$

To characterize this effective pressure, we first calculated the number of assembled wedges $n_{\mathrm{s}, P}$ in equilibrium simulations as a function of external hydrostatic pressure $P$ (see Supporting Information for simulation details). As expected, $n_{\mathrm{s}, P}$ increases monotonically with pressure, as illustrated in Supporting Figure 1a. In order to compare the degrees of assembly achieved under equilibrium conditions of constant $P$ and non-equilibrium driving with amplitude $a$, a relation $P(a)$ was established in the following way. We simulated a system of driven discs and undriven "ideal gas" particles. These particles interact with the driven species via a simple repulsive pair potential, but lack any mutual interactions. Due to the driving, the ideal gas particles segregate and form a compact cluster with surface area $S$ that depends on the amplitude of the driving $a$, as illustrated in Supporting Figure 1b. The effective surface pressure $P(a)$ can thus be determined from the ideal gas law, $P(a)=N k_{\mathrm{B}} T / S(a)$. In Figure 3b, we plot the number of assembled wedges $n_{\mathrm{s}, P(a)}$ at equilibrium conditions of constant $P$ parameterically as a function of the corresponding amplitude $a$. The resulting curve predicts similar degrees of assembly over the relevant range of amplitudes.

The precise dependence of $n_{s}$ on the driving $a$, however, can not be explained by analogy with equilibrium alone. To more accurately characterize the effective interactions between undriven particles, we have calculated effective potentials between two undriven particles in a bath of discs under the influence of "circular" shaking $\left(F_{x}=F \cos (2 \pi t / \tau)\right.$, $\left.F_{y}=F \sin (2 \pi t / \tau)\right)$ in Brownian dynamics simulations. The potentials are calculated as $u_{\tau, F}(r)=-k_{\mathrm{B}} T \ln \frac{p_{\tau, F}(r)}{p_{0}(r)}$, where $p_{\tau, F}(r)$ is the distribution function of the particle distance $r$ with shaking characterized by a period $\tau$ and amplitude $F$, and $p_{0}(r)$ is the corresponding equilibrium distribution for $F=0$. The distribution $p_{\tau, F}(r)$ was averaged over many periods of the external field. (In analogy with a potential of mean force for equilibrium systems, $u_{\tau, F}(r)$ can be interpreted as the interaction potential necessary to generate in the undriven system the $r$-statistics of the driven system. ${ }^{34}$ ) As illustrated in Figure $4 \mathrm{a}$, the associated forces are largely attractive and are consistent with the observed segregation. The strength and range of attraction increase with increasing period and amplitude. Interestingly, the potentials show modulations that depend on the frequency and amplitude of the driving force and reflect the structure of the "solvent" of biased particles.

The isotropic nature of averaged effective forces can be altered and directional forces between particles induced by applying suitable external fields. Figure $4 \mathrm{~b}$ shows the potential 

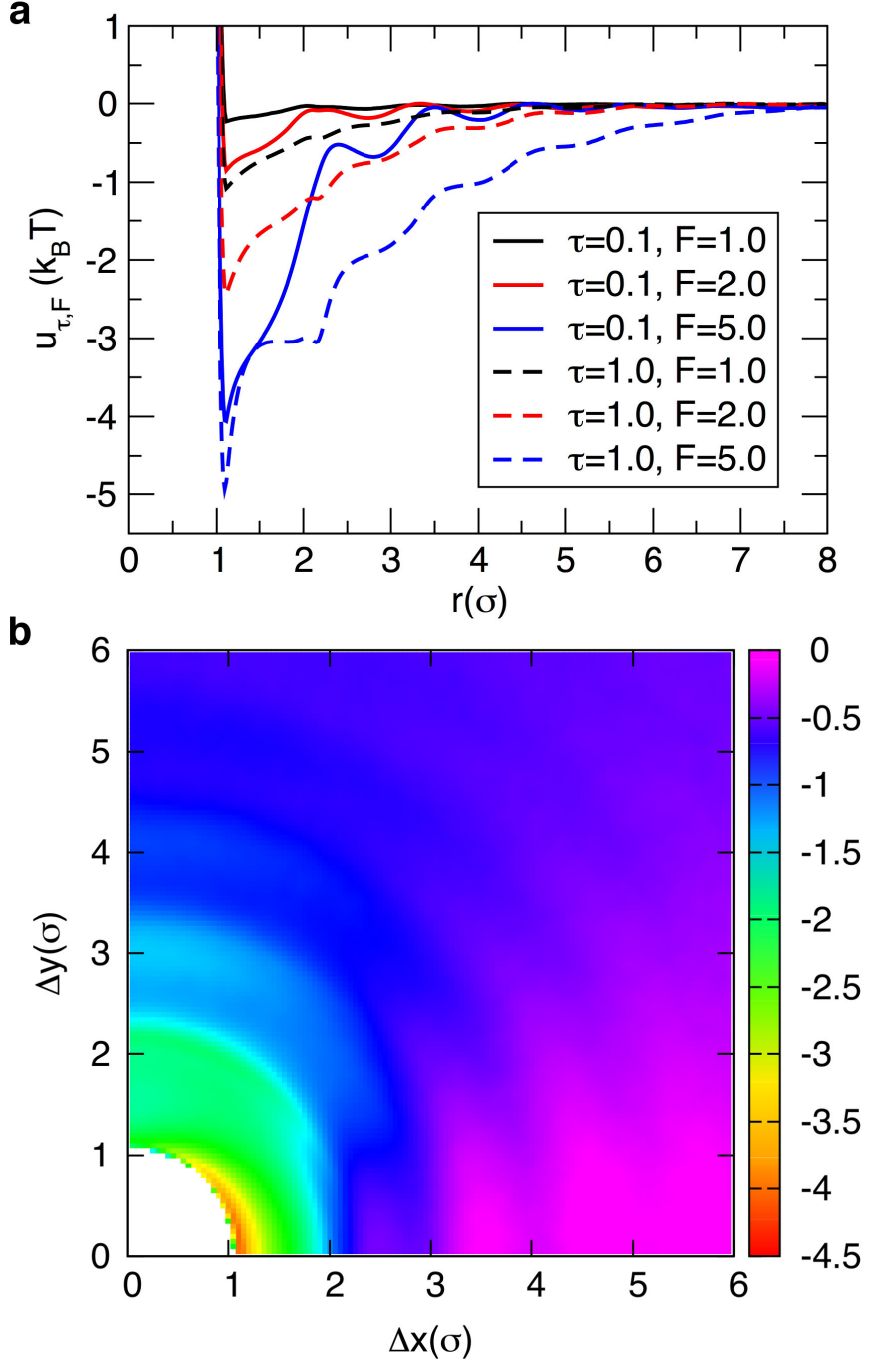

FIG. 4. Effective interactions from circular and uni-directional driving fields. (a) Effective interparticle potentials $u_{\tau, F}$ plotted as a function of the distance between two unbiased particles in a bath of particles driven by "circular" shaking, for different values of $\tau$ and $F$, in units of $\sigma \sqrt{m / \epsilon}$ and $\epsilon / \sigma$, respectively. (b) Effective interparticle potential (in $k_{\mathrm{B}} T$ ), as a function of the components $\Delta x$ and $\Delta y$ of the distance vector between two unbiased particles in a bath of particles driven by a uni-directional external force acting in the $x$-direction. Values of $u_{\tau, F} / k_{\mathrm{B}} T$ are indicated by color according to the scale at right.

of effective forces in a system driven by a uni-directional external force with components $F_{x}(t)=F \cos (2 \pi t / \tau)$ and $F_{y}=0$, with $F=5.0 \epsilon / \sigma$ and $\tau=1.0 \sigma \sqrt{m / \epsilon}$. The induced forces are strongly asymmetric in this case: The formation of dimers that are oriented in the direction of the field are favored by a few $\mathrm{k}_{\mathrm{B}} \mathrm{T}$ over the perpendicular orientation. While 
the attractive forces decay in $x$-direction within a few particle diameters, they act over a significantly longer range in the direction perpendicular to the driving.

Our observation of directional effective forces suggests that highly anisotropic patterns could be prepared that strikingly differ from typical equilibrium structures in self-assembling systems. To illustrate this possibility we concentrate on three-dimensional systems of particles with anisotropic shape, specifically plate-shaped particles and rods, as shown in Figure 5. Our simulations were initiated by placing hundreds of these particles in a dilute solution of spherical particles at a total packing fraction of 0.4. All particles interacted via purely repulsive, short-ranged pair potentials. A periodic external force was applied exclusively on spherical particles, and the Langevin dynamics of the system was followed at a temperature of $T=0.2 \epsilon / k_{\mathrm{B}}$ for $10^{5}$ units of simulation time. (Additional simulation details are given in the Methods section.)

For plates, we find unusual patterns under unidirectional shaking $\left(F_{x}(t)=F \operatorname{sgn}[\cos (2 \pi t / \tau)]\right.$, $\left.F_{y}=F_{z}=0\right)$. Our simulations show self-assembly of single-file columns that connect through the periodic boundary conditions in $x$-direction, as illustrated in Figure 5a. We note that the conditions under which we observe the formation of columns are roughly consistent with conditions at which "laning" is observed in mixtures of oppositely charged colloids in external electric fields. ${ }^{6}$ Similar string-like patterns have been observed in experiments and models of colloidal cubes under the influence of external electric fields. ${ }^{35}$

To corroborate that this highly anisotropic pattern is improbable under conditions of thermal equilibrium, we perform analogous simulations with zero nonthermal driving of spheres. In these equilibrium simulations of purely repulsive plates in a bath of spheres, stacks of plates do form transiently driven by weak depletion forces, but comprise only small numbers of plates, as illustrated in Figure 5b. In a second type of simulation, plates interact via short-range isotropic attractions of the Lennard-Jones type. In these simulations, columns of up to $\approx 10$ plates form in the early stages of assembly, but aggregate over time to form compact clusters, as illustrated in Figure 5c. Coarsening towards equilibrium proceeds very sluggishly in these simulations, and the completely equilibrated state (likely involving a columnar crystal of plates) could not be observed on the time scales accessible to simulation.

In the case of rods in a bath of driven spheres, we find formation of strikingly anisotropic patterns for "circular" shaking $\left(F_{y}(t)=F \cos (2 \pi t / \tau), F_{z}(t)=F \sin (2 \pi t / \tau), F_{x}=0\right)$. In particular, we observe self-assembly of a single column that consists of monolayer segments 
of close-packed rods, mostly aligned in the $x$-direction, perpendicular to the plane of driving, as illustrated in Figure 5d. As in the case of plates, such patterns do not occur in undriven systems. In simulations of purely repulsive rods at thermal equilibrium, nearby rods tend to align, but the typical size of clusters of aligned rods does not exceed a few particles (Figure 5e). In simulations with isotropic mutual attractions between rods, larger clusters of aligned rods form but aggregate over time in a non-specific manner (Figure 5f).

The forces driving pattern formation in our simulations are conceptually different from the effective forces responsible for the formation of liquid crystal phases in dense systems of hard rods and platelets. Nevertheless, filamentous patterns reminiscent of the one presented in Figure $5 \mathrm{f}$ have been observed in dense colloidal mixtures of rods and spheres, albeit under very different relative concentrations. ${ }^{36}$

\section{CONCLUSIONS}

Our experiments and simulations show that the effective forces associated with nonequilibrium phase separation of differentially driven particles can be manipulated to bias pattern formation within segregated phases. Experiments of agitated macroscopic shapes and computer models show that amplitude and frequency of periodic external forces control the degree of order observed in segregated phases, similar to the effect of external pressure in equilibrium systems. Highly anisotropic patterns can be achieved with strongly directional driving forces. In particular, simulations of rod- and plate-shaped particles indicate that binding motifs that occur transiently in equilibrium can be markedly amplified by suitable driving forces. Since particle attractions are effected by external biases only, assembled patterns can be easily un-assembled or modified by changing the driving force. This reversibility of interactions is in sharp contrast to the often irreversibly strong physical forces that hold together equilibrium structures of nanoparticles. A more detailed investigation of these principles could open up avenues to nanomaterials that more closely mimic the functionality and responsiveness of self-assembled structures in living systems. 

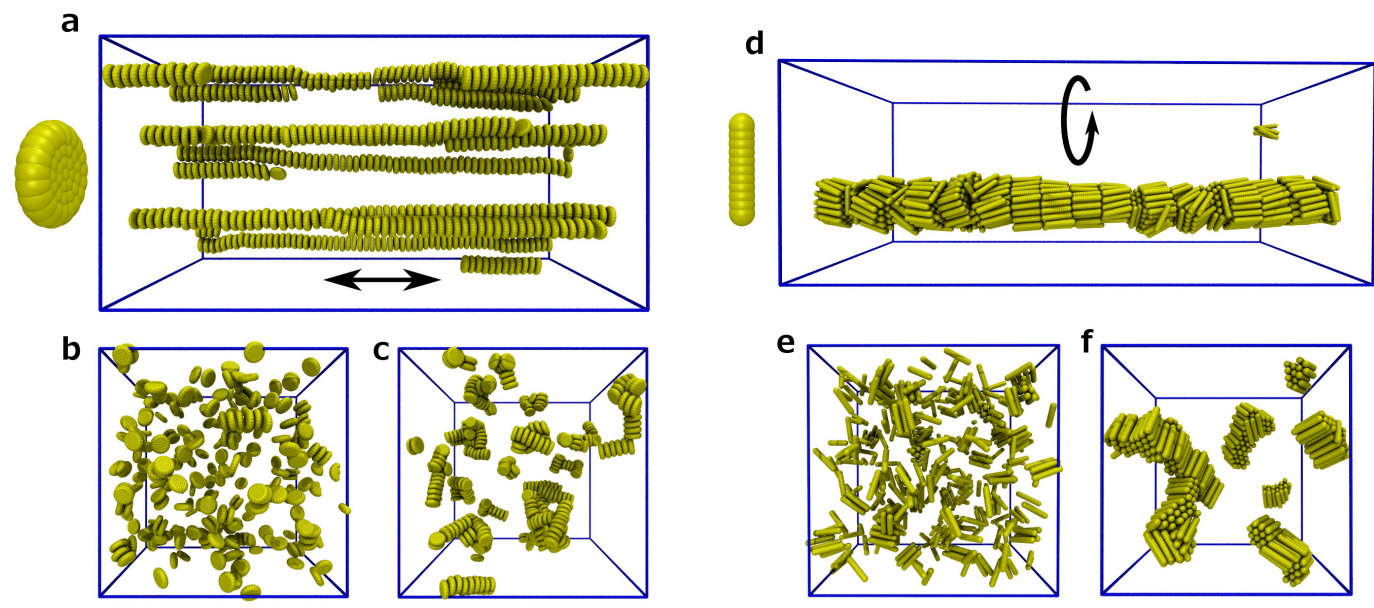

FIG. 5. Self-assembly of rods and plates into anisotropic patterns. (a) Snapshot of a system of rigid plates immersed in a bath of spheres (not shown) that are subject to a unidirectional periodic external force, as indicated by the arrows. The snapshot was taken after $10^{8}$ time steps of Langevin molecular dynamics that was initiated from a dispersed configuration of plates. The plates form single-file columns that connect through the periodic boundaries. (b) A typical equilibrium configuration of the same system without external forces. (c) A snapshot obtained after $10^{8}$ time steps of equilibrium simulation (spheres are not subject to external forces) in which plates not only exclude volume but also attract via isotropic Lennard-Jones potentials. (d) Snapshot of a system of rigid rods in a bath of spheres under circular shaking, as indicated by the arrow. Rods form a column that consists of monolayer-segments of tightly packed rods. (e) Equilibrium configuration of rods in the absence of external forces. (f) Attractive rods form disordered aggregates on computationally accessible time scales.

\section{METHODS}

\section{A. Experimental Setup}

\section{Materials and dimensions}

Star- and wedge-shaped prisms were cut from 3 mm thick PMMA sheets (McMaster Carr Supply Company) with a laser cutter (Universal Laser Systems, 50 Watt VLS 3.5). The base of wedges were equilateral triangles with $10 \mathrm{~mm}$ edge length. Stars had a diameter of 25 $\mathrm{mm}$, and pockets in the shape of equilateral triangles with edge lengths of $10 \mathrm{~mm}$ (for a 
perfect size match with the wedges) or $6.35 \mathrm{~mm}$ (for a size mismatch). Transparent PMMA beads of $6.35 \mathrm{~mm}$ diameter were purchased from Engineering Laboratories.

The board on which the objects were agitated had dimensions of $1.2 \mathrm{~m} \times 1.2 \mathrm{~m}$ and a mass

of $\approx 7 \mathrm{~kg}$ and was built from a planar aluminum honeycomb panel (purchased from Home Depot). The board was supported from the ceiling via an elastic cord joined to four cables anchored to the corners of the panel. The distance between the board and its attachment point on the ceiling was $1.3 \mathrm{~m}$. In the geometric center of the lower surface of the board, we attached a pendulum made from a rigid rod of $0.1 \mathrm{~kg}$ mass and $0.3 \mathrm{~m}$ length; to the end of the rod, we attached a weight of $1.4 \mathrm{~kg}$. The space accessible to shapes was restricted by an aluminum rim with a diameter of $0.48 \mathrm{~m}$. We covered the surface of the board with paper (Fadeless brand, purchased from Pacon Corp.). The roughness of the paper was chosen such that beads rolled easily under agitation, stars and the wedges, however, did not move unless hit by other solids. We filled the board with approximately 4100 beads, which corresponds to a packing fraction of 0.72. (A packing fraction of 0.91 corresponds to a hexagonal close-packed arrangement of discs in 2 dimensions.)

\section{Forces between plastic solids}

To ensure that interactions between solid particles were limited to collisions, we wrapped the experimental setup in a polyvinyl chloride (PVC) film to control the humidity above the plate. Using an air humidifier (Vicks V5100NS), we maintained a relative humidity larger than $60 \%$ around the experimental setup. Under these conditions, static electricity can be easily discharged and electrostatic interactions between solids are minimized. (See Supporting Information of Ref. 20.) Relative to forces of agitation, van der Waals forces between the solids in our experiments can be neglected.

\section{Agitation of the board and imaging}

To achieve pseudo-random agitation, we connected two motors to the board. An orbital shaker (Madell Technology Corp., ZD-9556-A) was attached to the board via an elastic polyurethane cord. The motion produced by the motor was a combination of orbital translation (with an amplitude of $5.1 \mathrm{~mm}$ ) and small-amplitude angular oscillation. The frequency 
was set to $120 \mathrm{rpm}$. To randomize this motion, we used as a second motor a linear actuator (LinMot Inc., P01-23x80) connected to the weight on the pendulum under the board. The actuator moved at a fixed frequency of $4 \mathrm{~Hz}$ in all experiments, and, in combination with the pendulum, lead to a irregular motion of the board. However, as confirmed by computer simulations described in this paper, segregation and assembly of shapes can be achieved through different modes of agitation. We repeated some experiments without the linear actuator in place and obtained qualitatively identical results. We took photos every 60 seconds using a Nikon D40 digital camera that was suspended $1 \mathrm{~m}$ above the plate.

\section{B. Simulation Details}

Input files for simulations performed in this work are available for download at http://gruenwaldgroup.com.

\section{Monte Carlo simulations of hard shapes}

Simulations comprised 218 discs with diameter $\sigma, 6$ equilateral triangular shapes (wedges) with edge length $\sigma$, and one star-shaped body, consisting of three rectangles with long-edge length $2.5 \sigma$ and short-edge length $\sigma /(2 \sqrt{3})$ (to achieve a pocket shape that matches the wedges, as shown in Fig. 1), or $\sigma / 2$ (for a size mismatch, as shown in Fig. 3), respectively. The packing fraction was set to 0.65 and periodic boundary conditions were used. Overlaps between shapes were detected using an algorithm based on the method of separating axis. ${ }^{37}$ Time is measured in "sweeps", each consisting on average of one attempt to displace each shape, and one attempt to rotate each anisotropic shape. The typical timescale for complete assembly of star and wedges from an initially dispersed state is millions of sweeps. In equilibrium simulations, components of particle displacements and rotations were drawn from uniform distributions with zero mean and widths of $0.01 \sigma, 0.03 \pi$, and $0.01 \pi$ for displacements of all shapes, rotations of wedges, and rotations of the star, respectively. To simulate shaking, the mean of the distribution of disc-displacements was modified as described in section II. $n_{\mathrm{g}}$ and $n_{\mathrm{s}}$ are defined as the time-averaged numbers of wedges within a distance $11 \sigma / 4$ and $\sigma[\sqrt{3} /(4 \sin (\pi / 3))+1 / 2]$ of the center of the star, respectively. All simulation snapshots were rendered with VMD. ${ }^{38}$ 


\section{Langevin dynamics of soft discs}

For simulations illustrated in Fig. 1e and Fig. 2, dynamics were advanced by numerically integrating the underdamped Langevin equation, as implemented in the HOOMD-blue simulation package ${ }^{39}$. For data in Fig. 1e, we simulated $N=2500$ particles in a periodically replicated square box at a packing fraction of 0.5. Particles interacted via the purely repulsive WCA potential. ${ }^{40}$ As basic units, we use the potential parameters $\sigma$ and $\epsilon$, and the particle mass $m$. We used friction coefficients of $2.0 \sqrt{m \epsilon} / \sigma$ and $1.0 \sqrt{m \epsilon} / \sigma$ for species $\mathrm{A}$ and $\mathrm{B}$,

respectively, a temperature of $T=0.1 \epsilon / k_{\mathrm{B}}$, and a time step of $\Delta t=0.001 \sigma \sqrt{m / \epsilon}$. All particles experienced an external periodic force characterized by $F=2.0 \epsilon / \sigma$ and $\tau=10 \sigma \sqrt{m / \epsilon}$. Data in Fig. 2 were obtained from systems of 900 particles at a packing fraction of 0.6 and a uniform friction coefficient of $10.0 \sqrt{m \epsilon} / \sigma$. Mean squared displacements were calculated from trajectories consisting of $2 \times 10^{7}$ timesteps.

Data presented in Figure 4 were obtained by integrating the overdamped Langevin equations of motion for 196 WCA particles at a packing fraction of 0.65, with a simple Euler-type integrator. ${ }^{41}$ We set $D \Delta t=0.0001 \sigma^{2}$, where $D$ is the diffusion coefficient, and $T=0.1 \epsilon / k_{\mathrm{B}}$.

\section{Langevin dynamics of three-dimensional shapes}

Dynamics were advanced by numerically integrating the underdamped Langevin equation, as implemented in the HOOMD-blue simulation package. ${ }^{39}$ Hydrodynamic interactions were neglected. Plates and rods were treated as rigid bodies consisting of spherical particles with diameter $\sigma$. Each plate comprised 40 such spheres. Spheres were arranged around a central sphere in three concentric conplanar rings with radii of $\sigma / 3,2 \sigma / 3$ and $\sigma$, comprising 7, 13, and 19 spheres, respectively. Discs therefore had an effective diameter of $3 \sigma$. Rods consisted of 13 spheres and had an effective length of $4.0 \sigma$. Simulations of plate assembly comprised 532 plates and 53,467 driven spheres of diameter $\sigma$. All spheres interacted via the WCA potential. ${ }^{40}$ We used a sphere friction coefficient $\gamma=10.0 \sqrt{m \epsilon} / \sigma$, a time step $\Delta t=0.001 \sigma \sqrt{m / \epsilon}$, and a box aspect ratio of 2:1:1. Assembly of columns was observed for $A=5.0 \sigma$ and $\tau=100.0 \sigma \sqrt{m / \epsilon}$. Simulations of rods involved 458 rods and 89,542 driven spheres in a box with aspect ratio 8:3:3. Assembly of columns was observed for $A=3.0 \sigma$ and $\tau=40.0 \sigma \sqrt{m / \epsilon}$. Equilibrium simulations were performed in cubic boxes. All simulations 
were performed at a packing fraction of 0.4 and a temperature of $T=0.2 \epsilon / k_{\mathrm{B}}$.

For the simulations illustrated in Figures $5 \mathrm{c}$ and $5 \mathrm{f}$, spheres in different rigid bodies interacted via the full Lennard-Jones potentials with $\epsilon_{\mathrm{rb}}=0.1 \epsilon$ and $\epsilon_{\mathrm{rb}}=0.2 \epsilon$, respectively. The temperature was $T=1.0 \epsilon / k_{\mathrm{B}}$.

\section{Acknowledgments}

We thank Meital Reches, Cristina Marchetti, and Christoph Dellago for useful discussions. MG's work was supported in part by the Austrian Science Fund (FWF) under Grant J 3106N16. The support and resources from the Center for High Performance Computing at the University of Utah are gratefully acknowledged. Calculations were in part performed on the Vienna Scientific Cluster (VSC). We acknowledge computational resources obtained under NSF award CHE-1048789. Research of ST and GMW was supported by the US Department of Energy (US DOE), Division of Materials Sciences and Engineering, under Award No. DEFG02-00ER45852. PLG was supported by the U.S. Department of Energy, Office of Basic

Energy Sciences, through the Chemical Sciences Division (CSD) of the Lawrence Berkeley National Laboratory (LBNL), under Contract DE-AC02-05CH11231.

\section{REFERENCES}

${ }^{1}$ S. Ramaswamy, Annu. Rev. Condens. Matter Phys. 1, 323 (2010).

${ }^{2}$ S. C. Warren, O. Guney-Altay, and B. A. Grzybowski, J. Phys. Chem. Lett. 3, 2103 (2012).

${ }^{3}$ M. E. Leunissen, C. G. Christova, A.-P. Hynninen, C. P. Royall, A. I. Campbell, A. Imhof, M. Dijkstra, R. van Roij, and A. van Blaaderen, Nature 437, 235 (2005).

${ }^{4}$ T. Vissers, A. van Blaaderen, and A. Imhof, Phys. Rev. Lett. 106, 228303 (2011).

${ }^{5}$ A. Wysocki and H. Löwen, Phys. Rev. E 79, 041408 (2009).

${ }^{6}$ T. Glanz and H. Löwen, J. Phys. Cond. Mat. 24, 464114 (2012).

${ }^{7}$ A. van Blaaderen, M. Dijkstra, R. van Roij, A. Imhof, M. Kamp, B. W. Kwaadgras, T. Vissers, and B. Liu, Eur. Phys. J. Spec. Top. 222, 2895 (2013).

${ }^{8}$ G. C. M. A. Ehrhardt, A. Stephenson, and P. M. Reis, Phys. Rev. E 71, 1 (2005).

${ }^{9}$ P. M. Reis, T. Sykes, and T. Mullin, Phys. Rev. E 74, 1 (2006). 
${ }^{10}$ G. D’Anna, P. Mayor, A. Barrat, V. Loreto, and F. Nori, Nature 424, 909 (2003).

${ }^{11}$ H. Jaeger, J. Knight, C.-H. Liu, and S. Nagel, MRS Bull. 19, 25 (1994).

${ }^{12}$ A. J. Olson, Y. H. E. Hu, and E. Keinan, Proc. Natl. Acad. Sci. U. S. A. 104, 20731 (2007).

${ }^{13}$ S. Tricard, E. Feinstein, R. F. Shepherd, M. Reches, P. W. Snyder, D. C. Bandarage, M. Prentiss, and G. M. Whitesides, Phys. Chem. Chem. Phys. 14, 9041 (2012).

${ }^{14}$ S. Tricard, C. A. Stan, E. I. Shakhnovich, and G. M. Whitesides, Soft Matter 9, 4480 (2013).

${ }^{15}$ S. Tricard, R. F. Shepherd, C. A. Stan, P. W. Snyder, R. Cademartiri, D. Zhu, I. S. Aranson, E. I. Shakhnovich, and G. M. Whitesides, Chempluschem 80, 37 (2015).

${ }^{16}$ G. K. Kaufman, M. Reches, S. W. Thomas, J. Feng, B. F. Shaw, and G. M. Whitesides, Appl. Phys. Lett. 94, 044102 (2009).

${ }^{17}$ B. A. Grzybowski, A. Winkleman, J. A. Wiles, Y. Brumer, and G. M. Whitesides, Nat. Mater. 2, 241 (2003).

${ }^{18}$ R. Cademartiri, C. A. Stan, V. M. Tran, E. Wu, L. Friar, D. Vulis, L. W. Clark, S. Tricard, and G. M. Whitesides, Soft Matter 8, 9771 (2012).

${ }^{19}$ I. Aranson and L. Tsimring, Rev. Mod. Phys. 78, 641 (2006).

${ }^{20}$ M. Reches, P. W. Snyder, and G. M. Whitesides, Proc. Natl. Acad. Sci. 106, 17644 (2009).

${ }^{21}$ Y. Mao, M. Cates, and H. Lekkerkerker, Physica A 222, 10 (1995).

${ }^{22}$ S. Asakura and F. Oosawa, J. Chem. Phys. 22, 1255 (1954).

${ }^{23}$ We speculate that depletion-induced assembly will eventually be observed under equilibrium conditions if beads are made smaller at constant packing fraction.

${ }^{24}$ M. E. Cates and J. Tailleur, arXiv:1406.3533.

${ }^{25}$ S. R. McCandlish, A. Baskaran, and M. F. Hagan, Soft Matter 8, 2527 (2012).

${ }^{26}$ M. Schnitzer, Phys. Rev. E 48, 2553 (1993).

${ }^{27}$ J. Tailleur and M. Cates, Phys. Rev. Lett. 100, 218103 (2008).

${ }^{28}$ F. D. C. Farrell, M. C. Marchetti, D. Marenduzzo, and J. Tailleur, Phys. Rev. Lett. 108, 248101 (2012).

${ }^{29}$ M. E. Cates and J. Tailleur, Europhys. Lett. 101, 20010 (2013).

${ }^{30}$ I. Buttinoni, J. Bialké, F. Kümmel, H. Löwen, C. Bechinger, and T. Speck, Phys. Rev. Lett. 110, 1 (2013).

${ }^{31}$ J. Bialké, T. Speck, and H. Löwen, J. Non. Cryst. Solids 407, 11 (2014). 
${ }^{32}$ L. Corté, P. M. Chaikin, J. P. Gollub, and D. J. Pine, Nat. Phys. 4, 420 (2008).

${ }^{33}$ A. P. Solon, J. Stenhammar, R. Wittkowski, M. Kardar, Y. Kafri, M. E. Cates, and J. Tailleur, Phys. Rev. Lett. 114, 1 (2015).

${ }^{34}$ J. Harder, S. A. Mallory, C. Tung, C. Valeriani, and A. Cacciuto, J. Chem. Phys. 141, 194901 (2014).

${ }^{35}$ H. R. Vutukuri, F. Smallenburg, S. Badaire, A. Imhof, M. Dijkstra, and A. van Blaaderen, Soft Matter 10, 9110 (2014).

${ }^{36}$ S. Fraden, M. Adams, Z. Dogic, and S. L. Keller, Nature 393, 349 (1998).

${ }^{37}$ P. Schneider and D. Eberly, Geometric Tools for Computer Graphics (Elsevier Science, 2002).

${ }^{38}$ W. Humphrey, A. Dalke, and K. Schulten, J. Mol. Graph. 14, 33 (1996).

${ }^{39}$ J. A. Anderson, C. D. Lorenz, and A. Travesset, J. Comput. Phys. 227, 5342 (2008).

${ }^{40}$ J. Weeks, D. Chandler, and H. C. Andersen, J. Chem. Phys. 54, 5237 (1971).

${ }^{41}$ P. Allen and D. Tildesley, Computer Simulation of Liquids (Clarendon Press, 1987). 